\title{
ABOUT SOME SCIENTIFIC PRINCIPLES OF VIBRO-IMPACT MACHINE DESIGN
}

\author{
Vladimir Astashev ${ }^{1}$, Vitaly Krupenin ${ }^{1,2}$ \\ ${ }^{1}$ Mechanical Engineering Research Institute of the Russian Academy of Sciences, Russia; \\ ${ }^{2}$ Moscow Polytechnic University, Russia \\ v_astashev@mail.ru, krupeninster@gmail.com
}

\begin{abstract}
Machines of vibro-impact type are widely used in agricultural and industrial production, being one of the most important subclasses of modern technological machines. These include traditional machines (rammers, crushers, vibro-hammers and many others), as well as modern ones, for example, ultrasonic technological devices and other important engineering objects. In most cases, these devices are designed without regard for the analysis of correct dynamic models, and the general theory of the organization of vibrating machines is far from complete. This makes it impossible to develop design schemes for similar facilities, to achieve the marginal utility of technological processes, and to ensure reliable and safe operation of machines. The vibro-impact machine design should be based on several universal scientific principles that have a clear engineering interpretation. First, we should mention the principle of resonance. When tuning the machine, as a rule, the working body must perform oscillations with maximum amplitude, since the maximum possible energy must be invested in the medium being processed. In addition, the machine realizes vibrational technological processes, which are mostly non-linear. Therefore, the problem of resonance tuning becomes nontrivial. Secondly, it is the principle of using arising nonlinear dynamic effects. Since vibro-impact machines are always non-linear objects, for which a sufficiently diverse specificity is characteristic, developers should use the manifested dynamic effects to improve their quality. The third principle is resource-saving. Vibro-impact machines should be designed in such a way that the costs for any resources for their creation and operation are reduced to the required minimum. The article articulates the above and some other principles that allow to create machines that satisfy the condition of marginal utility. The formulated principles are closely interrelated. When they are implemented, the vibro-impact machine will be used with maximum benefit.
\end{abstract}

Keywords: vibro-impact system, nonlinear resonance, vibration machine, linear system, strong nonlinearity.

\section{Introduction}

Vibro-impact machines are an important subclass of vibratory machines [1;2]. Numerous examples of such machines are used when immersed in the ground piles, pipes, dowels. They are used for tamping, compacting, loosening, and laying underground utilities. Crushing, grinding, mixing powders with their simultaneous grinding (homogenization) and other similar technologies are widely in demand in the chemical, food, construction, mining and other important industries. Vibratory crushers and screens are the basis of the mining industry. Vibro-impact molding of reinforced concrete products is successfully used in urban and rural construction in the construction industry; manual vibro-impact machines are widely used: they destroy strong soils, asphalt, concrete, break openings and niches, drill holes, clog crutches, etc. Hammers and perforators are necessary for drilling wells and boreholes.

Each such machine is defined by the triad "engine-oscillatory system-technological process". Almost all their known types are used as engines. Specificity of oscillatory systems is determined, mainly, by the design features and operating parameters. At the heart of each technological process there are dynamic phenomena that accompany systematic collisions of solids. The section of the theory of nonlinear oscillations, dealing with the study of these phenomena - "The theory of vibroimpact systems." The present article discusses some aspects of this theory for obtaining general scientifically based principles for designing vibro-impact machines.

\section{Vibro-impact systems}

Fig. 1 shows a standard scheme of a vibrating hammer [3] for immersion in the ground of piles, pipes, metal dowel. The system is a two-shaft vibrational exciter 1 with directed vertical vibrations, which drives the striker 3. Two electric motors are mounted in the exciter body, the parallel shafts of which synchronously rotate in different directions and are secured with unbalances 2 . The impact part and the lower plate 4 with the anvil 5 are interconnected by operating springs 6 . The headplate 7 is connected to the immersion element. When imbalances rotate, the vibrator thruster 3 generates frequent (up to tens of $\mathrm{Hz}$ ) impacts on the anvil 5, which is freely mounted on the lower hammer plate and transmits impacts directly to the immersion element. A widespread model of such a system - the 
so-called impact oscillator [4;5] - is given in Fig.1, b. Here, the parameters of the oscillator are determined by the design parameters of the vibration system of the vibration hammer, the parameters of the external force - the exciter.
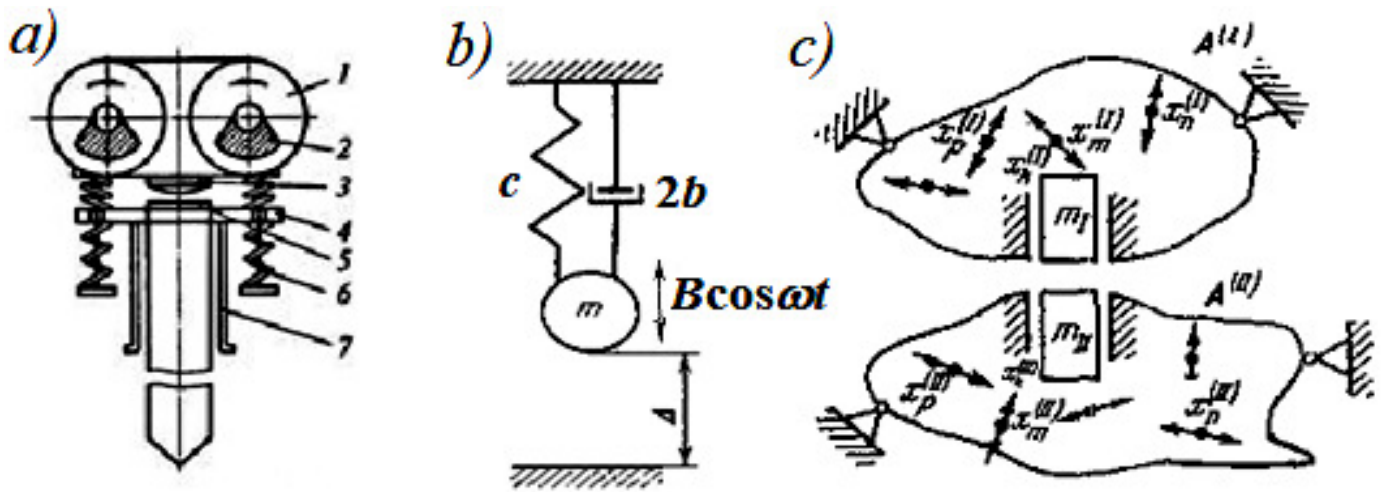

Fig. 1. Vibroimpact systems: a - vibro-hammer; $b$ - impact oscillator; $\mathrm{c}$ - general system

Fig. 1, c shows the scheme of a vibro-impact system of a general form: two interacting bodies through collisions with masses $m_{I}$ and $m_{I I}$, arbitrary linear stationary subsystems $A^{(I)}$ and $A^{(I I)}$. External forces are applied at some points $x_{k}^{(I, I)}$ to both subsystems.

The dynamics of the unified system is described by the operator equation

$$
u(t)=u_{0}(t)-L(i \omega) \Phi[u(t), i \omega u(t)],
$$

where $u(t)$ - unknown relative displacement of the colliding bodies;

$u_{0}(t)$ - this same displacement, but if the blows are neglected (the solution of the linear problem);

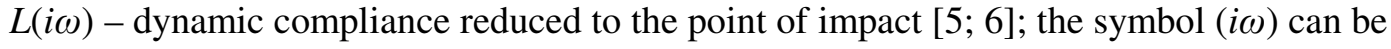
understood as the time differentiation operator;

$i^{2}=-1$;

$\Phi[u(t), i \omega u(t)]$ - force of impact.

If some collision occurs at a time $t=t_{k}$, and the impact is modeled according to Newton [5], then

$$
\Phi=-J \delta\left(t-t_{k}\right),
$$

where $J=(1+R) m\left|\dot{u}\left(t_{k}-0\right)\right|$ is the impact pulse;

$0<R \leq 1-$ recovery coefficient;

$m=m^{(I)} m^{(I I)}\left(m^{(I)}+m^{(I I)}\right)^{-1}$ is the reduced mass of colliding bodies;

$\delta(t)$ - the Dirac function.

Equation (1) can be investigated by frequency [5], time [7] or frequency-time methods [6]. The methods of the theory of vibro-impact systems are also described in [8-11]. We note that an equation similar to (1) was used in [12], when considering ultrasonic technological machines.

If we use frequency-time methods, then for the regime of motion with a period $T=2 \pi \omega^{-1}$ after substituting the representation of the force through the Dirac function into the formula (1), we can obtain:

$$
u(t)=u_{0}[t-\varphi(\omega)]-J(\omega) \sum L(i k \omega) \exp (i k \omega t) ; k=0, \pm 1, \pm 2, \ldots,
$$

where $\varphi(\omega)$-phase of vibro-impact mode.

For the problem of vibratory humming (Fig. 1, a) we can obtain:

$$
\begin{gathered}
L(i \omega)=\left[\left(\Omega_{1}{ }^{2}-\omega^{2}\right)+i 2 b_{1} \omega\right]^{-1} ; \\
u_{0}[t-\varphi(\omega)]=D(\omega) \cos (\omega t-\varphi) ; D(\omega)=B|L(i \omega)| ; \\
\Omega_{1}{ }^{2}=c m^{-1} ; b_{1}=b m^{-1} .
\end{gathered}
$$

Analyzing the solution (2), it is possible to obtain the amplitude-frequency characteristic of the vibro-impact system - the graph of the function $A(\omega)$, and $A$ is half of the maximum deviation of the 
body with the mass $m$ from the equilibrium position. For a general system (Fig.1, c), a qualitative picture is given by Fig. 2.

Unlike the standard amplitude-frequency characteristic of a linear system, one can see here the appearance of bends and characteristic branches of the curve. Fig. 2 denotes the eigenfrequencies of the combined linear system $A-\left\{\Omega_{k}\right\}$. Here $n$ is the number of degrees of freedom of the system A equal to the sum of the degrees of freedom of the subsystems $A^{(I)}$ and $A^{(I)}$. After the amplitude exceeds the value equal to the gap at the excitation frequency of the lower frequency of linear resonance, oscillations occur with collisions of small amplitude and intensity.

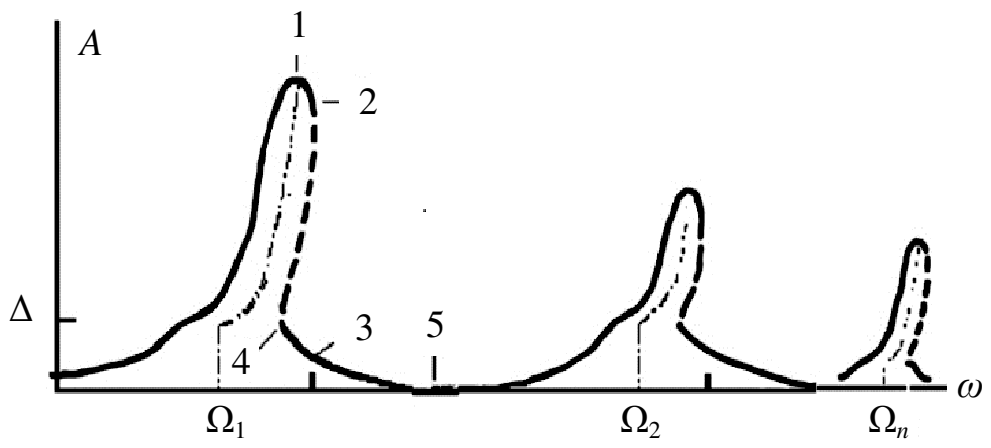

Fig. 2. Amplitude-frequency characteristic (AFC) of vibro-impact system

There are five characteristic points on the curve. Point 1 corresponds to the attainment of maximally large amplitudes; point 2 corresponds to a sudden termination of oscillations with collisions and an instantaneous transition to point 3 into a linear regime without interaction with the limiter; point 4 corresponds to a reverse transition to oscillations with collisions, which also appear "jump" after decreasing frequency; 5 - antiresonance point. We get here two-valued and even threevalued regimes. The dashed lines indicate physically unrealizable unstable regimes. In practice, it is possible to excite oscillations only near several first natural frequencies.

For the vibro-hammer model in the form of a system with one degree of freedom (Fig.1, b), the AFC remains qualitatively the same, but "higher maxima" will be absent. Point 1 in this case corresponds to the frequency $2 \Omega_{1}$. The amplitude-frequency response considered corresponds to the phenomenon of nonlinear resonance [4-6].

\section{Principle of resonant tuning}

The main requirement for any modern machine is high practical efficiency [12]. Therefore, the vibro-impact machine should produce the maximum possible energy E, which will be transmitted through the execution tool to the materials being processed with minimal losses.

The measure of this energy can be the amplitude of the vibrations of the working member $(A)$ or the impact pulse $(J)$. Here, the dependence of all quantities on the frequency of the regime is emphasized.

If the excitation produces a sinusoidal force, then using Fig. 2, one can conclude that the greatest energy is embedded in the medium at the resonance frequencies corresponding to point 1 and similar points in the region of higher frequencies. These frequencies can differ substantially from the frequencies of linear resonance, tuning of machines of vibro-impact action on which cannot be effective.

Thus, we arrive at an important principle that should be used as the basis for designing vibroimpact machines: the design of the machine must be determined by parameters that allow the machine to be tuned to nonlinear resonance. The values of these frequencies can be found with the aid of relation (2) and defined as a solution $\left(\omega=\omega_{1}, \ldots, \omega_{n}\right)$ of the frequency equation of the form:

$$
\Sigma L(i k \omega)=0 ; k=0, \pm 1, \pm 2, \ldots
$$

Details related to the solution of the frequency equation (3) can be found in the book [6].

It should be noted that in the nonlinear (vibro-impact) case the very concept of resonance is substantially complicated, and, in a certain sense, resonant frequencies are those occupying a certain 
interval or system of intervals. For a vibro-impact system with one degree of freedom, for example, this is an interval $\left(\Omega_{1}, 2 \Omega_{1}\right)$. The value of the amplitude at the frequency $2 \Omega_{1}: A=A^{*} \approx A\left(2 \Omega_{1}\right)$ turns out to be maximally possible for the given characteristics of the drive and the energy dissipation levels for the processing of any medium: all physically realizable amplitudes, if we consider the parameter $A \leq A^{*}$. If we consider the parameter

$$
\vartheta=\frac{A}{A^{*}} \times 100 \%
$$

called the coefficient of practical efficiency, it can be established that, for example, for resonant ultrasonic technological machines, also belonging to the class of vibro-impact machines, limit possibilities can be realized, when the efficiency value in the classical sense is $50 \%([13 ; 14])$.

\section{Stabilization of resonant modes}

A distinctive feature of the realization of nonlinear resonant regimes is their instability. From the graph of Fig. 2: when the frequency deviates to the right from the value corresponding to the maximum possible amplitude, the effective vibro-impact mode is disrupted. So we come to another important principle.

When designing a resonant vibro-impact machine, it is necessary to provide a stabilization system for the resonance regime. Such a system should ensure that oscillations are held near point 1 of the amplitude-frequency characteristic, Fig. 2. This can be done by help of various types of automatic frequency control systems, but the most effective is the organization of an autoresonant excitation scheme for a vibro-impact machine: Fig. 3 [13; 14].

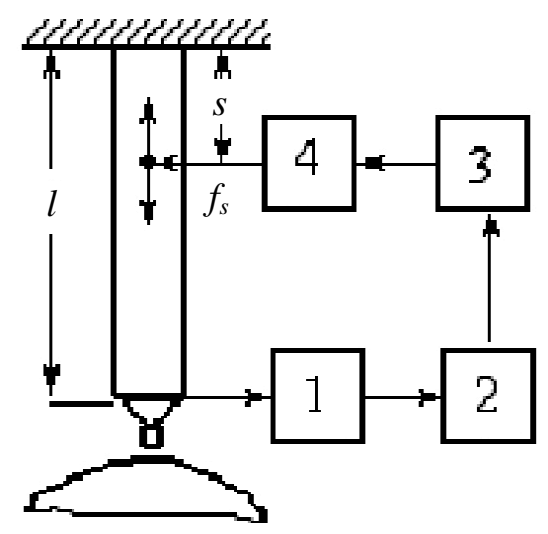

Fig. 3. Scheme of autoresonance machine

The oscillatory system is, for example, a viscoelastic rod of length 1 and performs longitudinal oscillations under the action of the force $f_{s}$ applied in a certain section $x=s$. As a result, vibration is transferred to the treatment zone, where a vibro-impact process is initiated.

The exciting force is created by means of a chain of feedback around the rod. It contains a sensor 1 detecting the movement of the cross-section $x=1$, an amplifier 2, a phase changer 3 and a transducer 4 creating a force action. At a certain phase of the feedback signal, which is set by the phase changer, resonance oscillations are excited, the frequency of which depends on the technological load.

If the phase shift is chosen so that the phase difference between the oscillations of the tool and the force produced by the exciter corresponds to a resonant value, then the device will ensure the realization of the resonant modes, when the load and the parameters of the oscillatory system vary in a wide range.

The described system makes it possible to realize all stable branches of the amplitude-frequency characteristic (Fig. 2) in the self-oscillation mode and to provide stable vibro-shock modes corresponding to point 1 . At this point (it is also called the boundary point of conditions for the existence of resonant regimes), the AFC merges with a dotted line corresponding to movement, which could exist without considering the forces of friction and excitation. 
Thus, the organization of schemes for autoresonant excitation of vibro-impact processes makes it possible to maximize the practical efficiency of the technological process with a minimum of possible energy costs. Therefore, such a scheme corresponds to the functioning of the machine in the mode of maximum energy efficiency.

This agrees with one more important principle of designing modern technological machines "maximum efficiency with minimum energy consumption".

\section{Nonlinear dynamic effects}

From the point of view of modern nonlinear mechanics, vibro-impact machines turn out to be strongly nonlinear dynamic objects, since the non-linear factors introduced by collisions have determining influences on the characteristics of motion.

Knowledge and consideration of the dynamic effects accompanying the operation of these machines and manifested due to the presence of strong ("impact") nonlinear forces constitute the essence of a very important principle in the design of machines of this type.

Let us give a brief description of such effects, which can appear in each nonlinear system, but are especially evident for vibro-impact systems.

1. Generation of higher harmonic components. Vibro-impact processes have very representative spectra. Using formula (2), it can be shown that higher harmonics can have a decisive influence on the behavior of systems.

2. The dynamic phenomena of frequency and amplitude pulling. To obtain intensive vibro-impact regimes (point 1 on the amplitude-frequency characteristic of Fig. 2), we can smoothly increase the excitation frequency after passing through linear resonances (the effect of frequency pulling). Impulse of impact is proportional to the size of the gap. Smoothly increasing the gap, you can achieve an increase in the amplitude of vibration and the intensity of the vibro-impact mode (the effect of amplitude pulling).

3. Downward jump and hard start. As noted, after passing through the boundary conditions for the existence of resonant regimes, a sudden decrease in the amplitude of the oscillations (from point 2 to point 3 of the AFC) occurs -"downward jump". Return to resonant vibro-impact modes is possible only after additional impact at a frequency that is lower than the frequency of downward jump (the possibility of a hard start). Return without a hard start is possible only after a significant decrease in the frequency value (point 4).

4. Subharmonic and combination resonance regimes. Modes of motion with frequency $\omega l^{-1}$ can exist due to the action of forces of a higher frequency $\omega$, and $l$-natural number (subharmonic resonant modes $1: l)$. When these modes occur, the first harmonic of the external force does the work on the $l$-th harmonic of the solution, which can be written in the form (2); the second on the $2 l$-th, and so on. In the presence of a periodic external force containing a large number of higher harmonics, it is possible to have combination resonance regimes with a frequency $\omega p l^{-1}$. Such modes are characterized by the fact that the work of the $p$-th harmonic of the external force is done at the $l$-th harmonic of motion.

5. Almost periodic and chaotic regimes; "chatter". In some cases, the vibro-impact modes of motion lose their stability and then there are complicated vibro-impact regimes: almost periodic (their spectrum consists of harmonics with incommensurable frequencies); chaotic with a continuous spectrum. Near the frequencies of linear resonance, regimes with many impacts during the period of motion (so-called "chatter" modes) are also possible. Note that in the study of such regimes it is most often necessary to exclude their occurrence: effective resonant regimes are "correct" in nature.

Fig. 4 shows a schematic diagram of a device for carrying out vibration tests. The device reproduces the broadband vibro-impact process by the natural method [15]. Three modules with hammers tuned to subharmonic resonance modes are mounted on the vibrating platform. The impact is transferred to the desktop, where the test object is installed. The device is configured using the calculation formula (2) written for this task, as well as the vibration test program. The locations of the modules are also calculated. An auxiliary device is added to the system, which allows to provide a 
hard start, for example, shaking in the event of accidental disruption of resonant subharmonic regimes. It is possible to organize the operation of the system using the principles of autoresonance.

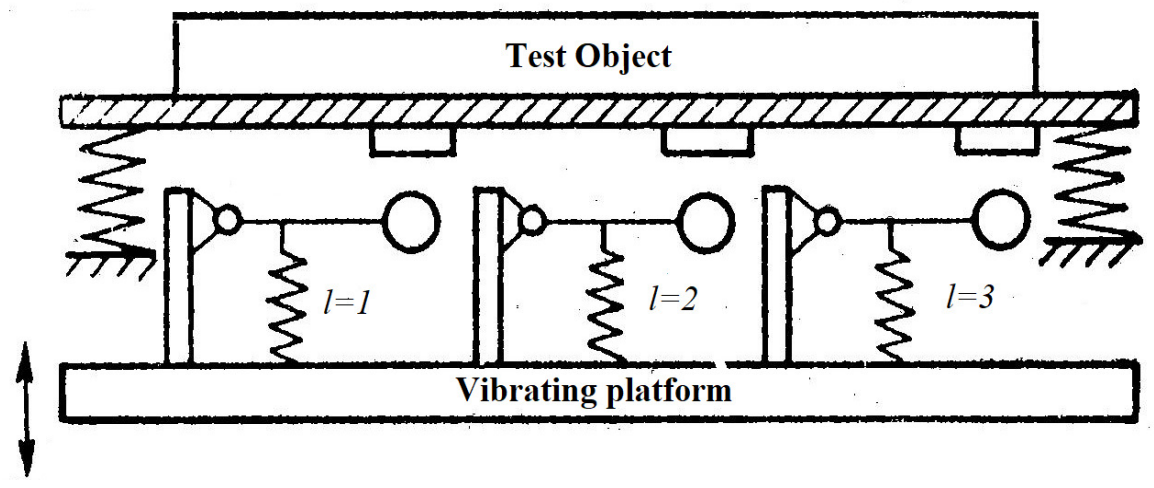

Fig. 4. Resonance vibro-impact test system

\section{Conclusions}

Vibrating impact machines are strongly nonlinear technical objects, in which numerous specific dynamic effects manifest themselves. The study and consideration of these effects are necessary at the design stage. Information about them should be used in the design of high-performance and resourcesaving systems, and to implement their current tuning to the regimes of maximum practical efficiency. The main object of attention is resonant vibro-impact modes of motion.

\section{Acknowledgements}

This work was supported by the Russian Foundation for Fundamental Research (project 18-0800168).

\section{References}

[1] Вибрации в технике: Справочник. Т.4. Вибрационные машины и процессы. (Vibration in Engineering: Handbook. Vol.4. Vibration machines and processes) Lavendelis E.E (ed.), Moscow: Mashinostroenie, 1981. 510 p. (In Russian).

[2] Бабицкий В. И., Крупенин В.Л. Машины ударного действия. (The Impact Action Machines). Moscow: Znanie. 1987. 62 p. (In Russian).

[3] Кожевников С.Н., Есипенко Я.И., Раскин Я.М. Механизмы. (The mechanisms.) Handbook. Moscow: Mashinostroenie, 1976. 784 p. (In Russian).

[4] Асташев В.К. К динамике осциллятора, ударяющегося об ограничитель. (To the dynamics of the oscillator impacting the limiter). Mashinovedinie, 1971. No 2, pp. 5-9. (In Russian).

[5] Babitsky V. I. Theory of Vibro-Impact Systems and Applications. Berlin: Springer-Verlag, 1998. $318 \mathrm{p}$.

[6] Babitsky V.I., Krupenin V.L Vibration of Strongly Nonlinear Discontinuous Systems. Berlin: Springer-Verlag, 2001. $404 \mathrm{p}$.

[7] Кобринский А.Е. Механизмы с упругими связями. (Mechanisms with elastic bonds). Moscow: Nauka, 1964. 392 p. (In Russian).

[8] Вибрации в технике: Справочник. Т.2. Колебания нелинейных механических систем. (Vibration in Engineering: Handbook. Vol.4. Vibration of nonlinear mechanical systems.) Blechman I.I. (ed.), Moscow: Mashinostroenie, 1979. 352 p. (In Russian).

[9] Viba J., Lavendelis E. Algorithm of synthesis of strongly non-linear mechanical systems. In Industrial Engineering - Innovation as Competitive Edge for SME, 22 April 2006. Tallinn, Estonia, pp. 95-98.

[10] Ibrahim R.A. Vibro-Impact Dynamics: Modeling, Mapping and Applications. Berlin: SpringerVerlag, 2009. $312 \mathrm{p}$.

[11]Luo A.C.J., Guo Y. Vibro-impact Dynamics. Berlin: Springer-Verlag, 2013. 213 p.

[12] Astashev V., Krupenin V. Efficiency of vibration machines, Engineering for Rural Development. Jelgava, Latvia, 2017, pp.108-113. 
[13] Асташев В.К., Крупенин В.Л. Нелинейная динамика ультразвуковых технологических процессов (Nonlinear Dynamics of Ultrasonic Technological Processes). Moscow: MPU(MGUP), 2016. 372 p. (In Russian).

[14] Astashev V.K., Babitsky V.I. Ultrasonic Processes and Machines. Dynamics, Control and Applications. Berlin: Springer-Verlag, 2007. 330 p.

[15] Veprik A.M. and other Широкополосные виброударные генераторы механических колебаний (Broadband vibro-impact generators of mechanical oscillations). Leningrad: Mashinostroenie, 1987. 87 p. (In Russian). 